\title{
Métodos de avaliação da distribuição longitudinal de plântulas no estabelecimento inicial da cultura do milho
}

\author{
Paula Cristina Natalino RINALDI ${ }^{1 *}$, Renan ZAMPIROLI ${ }^{1}$, Cleyton Batista de ALVARENGA ${ }^{1}$, \\ Rodrigo Bezerra de Araújo GALLIS ${ }^{1}$, Haroldo Carlos FERNANDES² \\ 1 Instituto de Ciências Agrárias, Universidade Federal de Uberlândia, Monte Carmelo, MG, Brasil. \\ 2 Departamento de Engenharia Agrícola, Universidade Federal de Viçosa, Viçosa, MG, Brasil. \\ *E-mail: paularinaldi@ufu.br \\ (Orcid: 0000-0001-9290-9040; 0000-0002-5082-9111; 0000-0002-0431-6171; 0000-0002-8764-9173; 0000-0001-5276-5441)
}

Recebido em 08/04/2020; Aceito em 26/03/2021; Publicado em 23/04/2021.

RESUMO: A velocidade de trabalho é um dos fatores que pode influenciar a distribuição longitudinal de plântulas, o estande final e a produtividade da lavoura. Objetivou-se com este trabalho avaliar, em condições de campo, o desempenho de uma semeadora-adubadora no estabelecimento inicial da cultura do milho, em quatro velocidades de trabalho e dois mecanismos sulcadores por meio de dois métodos de medição entre plântulas. O delineamento experimental foi em blocos casualizados, com quatro repetições, combinando velocidades de trabalho e mecanismos sulcadores para deposição das sementes. A avaliação da distribuição longitudinal de plântulas se deu por dois métodos, manual e imagens captadas por aeronave remotamente pilotada. $\mathrm{O}$ aumento da velocidade de trabalho influenciou o percentual de espaçamento aceitáveis entre plântulas na velocidade de $6 \mathrm{~km} \mathrm{~h}^{-1}$ para a deposição da semente pelo sulcador de disco, método manual de avaliação. As variáveis relacionadas ao espaçamento entre plântulas não diferiram com o aumento da velocidade de trabalho do conjunto mecanizado e nem com a variação do sulcador pelo método de avaliação por imagem. A velocidade de trabalho não influenciou o índice de velocidade de emergência de plântulas, sendo este maior com o uso da haste.

Palavras-chave: velocidade de trabalho; mecanismo sulcador; semeadora.

\section{Methods of evaluation longitudinal distribution of seedlings in the initial establishment of maize crops}

\begin{abstract}
The working speed is one of the factors that can influence the longitudinal distribution of seedlings, the final stand and the productivity of the crop. The aim of the current study is to assess, under field conditions, the performance of a seed drill/fertilizer machine in the initial establishment of maize crops, according to four working speeds and two furrowing mechanism using two seedlings measurement methods. The experiment followed a randomized block design with four repetitions; it combined working speeds and furrowing mechanisms for seed deposition. The evaluation longitudinal distribution of seedlings of two methods, manual and images obtained with unmanned aerial vehicle. The increase in the working speed influenced the percentage of acceptable spacing between seedlings at the speed of $6 \mathrm{~km} \mathrm{~h}^{-1}$ for the deposition of the seed by the mismatched double disk-type furrow, manual method of evaluation. The variables related to spacing between seedlings did not differ with the increase in the working speed of the mechanized set without variation of the furrow by the image evaluation method. The working speed did not influence the seedling emergence speed index, being higher with furrow blade.

Keywords: working speed; furrow mechanism; seeder.
\end{abstract}

\section{INTRODUÇÃO}

A eficácia da semeadura é um dos fatores que mais afeta a produtividade de uma cultura, requerendo assim, precisão e bom controle da operação (CARPES et al., 2018). Nesse sentido, a uniformidade de distribuição de sementes e a velocidade de semeadura estão diretamente relacionadas à qualidade de implantação de uma lavoura e, consequentemente, o estande de plantas obtido influenciará diretamente a produtividade da cultura do milho (BOTTEGA et al., 2018).

Tal fato foi demonstrado por Ormond et al. (2018), operando nas velocidades de 2,0;4,7; 6,5; 9,1 e 10,3 $\mathrm{km} \mathrm{h}^{-1}$, com os melhores percentuais de espaçamentos aceitáveis nas velocidades até $6,5 \mathrm{~km} \mathrm{~h}^{-1}$, indicando que o aumento da velocidade pode acarretar aumento dos espaçamentos falhos e múltiplos para a cultura do milho, comprometendo a eficácia da semeadura. Carpes et al. (2017) verificaram que, com o aumento da velocidade periférica do disco dosador de sementes, há uma tendência de redução no nível de precisão de deposição das sementes na linha de semeadura, diminuindo os espaçamentos aceitáveis entre plântulas.

As metodologias tradicionais para avaliação do desempenho de semeadoras e suas variações baseiam-se nos espaçamentos, medidos in loco no campo, entre as plântulas e classifica os espaçamentos em aceitáveis, falhos ou duplos. Porém, com o advento de novas tecnologias que facilitam a 
mensuração desses espaçamentos pretende-se avaliar a possibilidade dessa mensuração por imagens captadas a partir de aeronave remotamente pilotada.

O sensoriamento remoto vem ocupando cada vez mais aplicação como ferramenta de medida e avaliação em diversas culturas agrícolas. Um exemplo é na contagem de plantas por meio de aeronaves remotamente pilotadas, do qual em um curto intervalo de tempo permite obter o número de indivíduos com precisão, permitindo assim, por meio de identificação das falhas de plantio, realizar um planejamento para o replantio (Arantes et al., 2019), garantindo uniformidade de estande de plantas.

Stolf et al. (2016) avaliando dois procedimentos para determinação de falhas em cana-de-açúcar, aéreo (aeronave remotamente pilotada) e terrestre (convencional manual), verificaram consistência dos resultados no tamanho médio e frequência de aparecimento de falhas entre o procedimento padrão e o imageamento aéreo, indicando validade da proposta do emprego de imagens de alta definição.

Diante do exposto, objetivou-se avaliar, a uniformidade do espaçamento entre plântulas, produzido por uma semeadora-adubadora no estabelecimento inicial da cultura do milho, em diferentes velocidades de trabalho e tipos de mecanismos sulcadores por meio de métodos terrestre e aéreo.

\section{MATERIAL E MÉTODOS}

O experimento foi conduzido em área pertencente à Universidade Federal de Uberlândia, localizada no município de Monte Carmelo - MG. A localização geográfica da área, está definida pelas coordenadas $18^{\circ} 42^{\prime} 43,19^{\prime \prime} \mathrm{S}$ e $47^{\circ} 29^{\prime} 55,8^{\prime}$ ” W, com uma altitude de 873 metros. O relevo da região é $20 \%$ montanhoso, $60 \%$ ondulado e $20 \%$ plano. O clima, conforme a classificação de Köppen (1948), é denominado Aw (clima tropical com estação seca de inverno).

A área foi dessecada com glyphosate, sal de amônio de glifosato na dose de 1,3 $\mathrm{L} \mathrm{ha}^{-1}$ (792,5 g i.a kg-1), volume de calda de $250 \mathrm{~L} \mathrm{ha}^{-1}$. Em campo, utilizou uma semeadoraadubadora, marca Vence Tudo, modelo SA 11500 A, montada no levante hidráulico de três pontos, um trator marca Valtra, modelo A850, tração 4x2 com tração dianteira auxiliar (TDA), potência nominal de $85 \mathrm{cv}(62,5 \mathrm{~kW})$, rotação de $2.300 \mathrm{rpm}$ no contagiros, motor com $3.300 \mathrm{~cm}^{3} \mathrm{de}$ cilindrada total e peso máximo permitido $4.675 \mathrm{kgf}$.

A semeadora foi equipada com mecanismos dosadores de sementes do tipo disco alveolado horizontal, disco de corte de palhada liso de 16" de diâmetro, sulcadores para deposição de sementes de discos duplos defasados de 13" de diâmetro e hastes sulcadoras, largura de $0,03 \mathrm{~m}$.

O experimento foi conduzido no delineamento de blocos ao acaso em esquema fatorial $4 \times 2$; (sendo quatro velocidades de trabalho do conjunto mecanizado trator-semeadora e dois mecanismos sulcadores); totalizando 8 tratamentos, com quatro blocos (Tabela 1).

As parcelas apresentaram as dimensões de 1,5 metros de largura por 30 metros de comprimento, com área de $45 \mathrm{~m}^{2}$; entre as parcelas deixou-se uma distância de 1,8 metros; e foi desconsiderada os dez primeiros metros de cada extremidade, a fim de que a avaliação não fosse influenciada pela estabilização das velocidades do conjunto mecanizado.

A semeadora-adubadora foi regulada para um espaçamento entre as linhas de $0,50 \mathrm{~m}$, depositando cinco sementes por metro linear, espaçadas de 0,20 metros entre si, na profundidade de $0,05 \mathrm{~m}$, o lote de sementes apresentava germinação de $98 \%$, pureza de $98 \%$, vigor de $89 \%$, e um estande desejado de 85.000 plantas por hectare. A semeadora trabalhou com quatro linhas, sendo consideradas úteis as duas centrais e as duas da extremidade foram consideradas bordadura.

Tabela 1. Marchas do trator em função da velocidade de trabalho do conjunto mecanizado

Table 1. Tractor marches in function of the working speed mechanized sit

\begin{tabular}{cccc}
\hline $\begin{array}{c}\text { Velocidade } \\
\left(\mathrm{km} \mathrm{h}^{-1}\right)\end{array}$ & Mecanismo sulcador & Marcha & $\begin{array}{c}\text { Rotação } \\
(\mathrm{rpm})\end{array}$ \\
\hline 6 & Disco duplo defasado & $\mathrm{L} 4$ & 1.800 \\
8 & Disco duplo defasado & $\mathrm{H} 1$ & 2.100 \\
10 & Disco duplo defasado & $\mathrm{H} 2$ & 2.000 \\
12 & Disco duplo defasado & $\mathrm{H} 3$ & 1.600 \\
6 & Haste sulcadora & $\mathrm{L} 4$ & 1.950 \\
8 & Haste sulcadora & $\mathrm{H} 2$ & 1.650 \\
10 & Haste sulcadora & $\mathrm{H} 2$ & 2.100 \\
12 & Haste sulcadora & $\mathrm{H} 3$ & 1.600 \\
\hline
\end{tabular}

Os parâmetros espaçamento entre sementes; distribuição longitudinal entre plântulas, espaçamentos aceitáveis, falhos e múltiplos; índice de velocidade de emergência e tempo médio de emergência de plântulas foram utilizados para inferir sobre a qualidade da semeadura.

O experimento foi dividido em duas etapas: contagem das plântulas do primeiro dia de emergência até a estabilização, a fim de determinar o índice de velocidade de emergência e o tempo médio de emergência; e a segunda etapa, após a estabilização de emergência das plântulas, mediu-se o espaçamento entre plântulas para determinar a distribuição longitudinal, enquadrando-os em aceitáveis, falhos e múltiplos, por dois métodos de avaliação, manual e imagens captadas por aeronave remotamente pilotada. As imagens foram obtidas dez dias após a contagem pelo método manual para adequar o estádio de desenvolvimento à qualidade das imagens para diferenciação das plântulas.

A contagem das plântulas, método manual, iniciou no primeiro dia de emergência, quatro dias após a semeadura, em uma faixa de dez metros no centro da parcela, sendo a contagem feita nas duas linhas úteis em todas as parcelas e encerrada após as plantas atingirem a estabilização da emergência, que ocorreu nove dias após a semeadura.

A determinação do índice de velocidade de emergência de plântulas (IVE) foi realizada pelo somatório da relação entre o número de plantas emergidas (primeira, segunda, até a última contagem) pelo número de dias da semeadura (primeira, segunda, até a última contagem), utilizando-se metodologia de Maguire (1962), (Equação 1).

$$
I V=\frac{E_{1}}{T_{1}}+\frac{E_{2}}{T_{2}}+\ldots+\frac{E_{n}}{T_{n}}
$$

em que: IV = índice de velocidade emergência; $E_{1 \ldots n}=$ número de plântulas emergidas na primeira, segunda até a última contagem; $T_{1 \ldots n}=$ número de dias da semeadura à primeira, segunda até $\mathrm{a}$ última contagem.

O tempo médio de emergência (TM), em dias, foi calculado de acordo com Edmond; Drapala (1958). Para isso, multiplicou-se o número de plântulas emergidas desde a primeira contagem pelo tempo médio de emergência (dias), posteriormente dividiu-se o valor encontrado pelo somatório 
do número de plântulas emergidas desde a primeira contagem (Equação 2).

$$
N_{m}=\frac{E_{1} \cdot T_{1}+E_{2} \cdot T_{2}+\ldots+E_{n} \cdot T_{n}}{E_{1}+E_{2}+\ldots+E_{n}}
$$

em que: $\mathrm{N}_{\mathrm{m}}=$ tempo médio de emergência, em dias; $E_{1 \ldots . n}=$ número de plântulas emergidas desde a primeira contagem; $T_{1 \ldots n}=$ número de dias após a semeadura.

A distribuição longitudinal entre plântulas foi obtida por dois métodos, manual e por meio de imagens captadas por Aeronave Remotamente Pilotada - ARP.

O método manual foi obtido medindo-se o espaçamento entre vinte plântulas, nas duas linhas centrais de semeadura, logo após estabilização da emergência. Posteriormente, foram classificados os espaçamentos em aceitáveis ( $0,5^{*} \mathrm{Xref}<\mathrm{Xi}<1,5^{*} \mathrm{Xref}$ ), falhos $\left(\mathrm{Xi} \geq 1,5^{*} \mathrm{Xref}\right)$ e múltiplos (Xi $\leq 0,5^{*} \mathrm{Xref}$ ), de acordo com International Organization for Standardization - ISO 7256-1 (1984), metodologia também utilizada por Bottega et al. (2018), Carpes et al. (2018), Cortez et al. (2020), Ferreira (2019), Klein; Klein (2018).

Os valores foram expressos em porcentual, calculados sobre o número total verificado. Considerando que a semeadora foi regulada para distribuir cinco sementes $\mathrm{m}-1$, o espaçamento referência (Xref) corresponde a 0,20 m e, desta forma, foram considerados como aceitáveis, espaçamentos de 0,10 a $0,30 \mathrm{~m}$ entre plântulas; falhos maiores que $0,30 \mathrm{~m} \mathrm{e}$ múltiplos menores que $0,10 \mathrm{~m}$.

Para determinar o espaçamento entre plântulas, por meio de imagens, realizou-se um voo planejado para obtenção de uma ortoimagem com GSD (Ground Sample Distance), com resolução espacial de $1 \mathrm{~cm}$. O voo foi realizado com uma aeronave remotamente pilotada - ARP, marca DJI, modelo Phantom 4 Pro com câmara RGB de 20 megapixel. Após o voo, de posse do conjunto de imagens da área de estudo, realizou-se o processamento das imagens no software Pix4D para geração da ortoimagem (Figura 1).

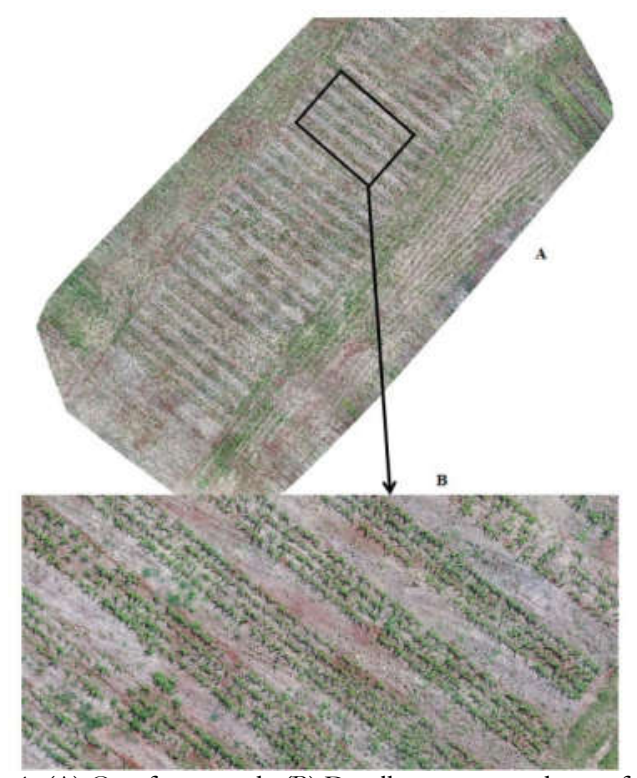

Figura 1. (A) Ortofoto gerada (B) Detalhe em zoom da ortofoto. Figure 1. (A) Generated orthophoto (B) Zoom detail of the orthophoto.
A contagem da distância, centro a centro, entre plântulas foi realizada na mesma faixa estabelecida para contagem de plântulas pelo método manual. As medidas das distâncias foram realizadas com auxílio do software Quantum GIS, utilizando a ferramenta de media linear existente no software. A Figura 2 exemplifica o processo de medição. Um processamento invertendo os canais verde $(G)$ pelo azul (B) foi realizado para inverter as cores das plântulas de modo a realçá-las a fim de possibilitar o processo de medição.

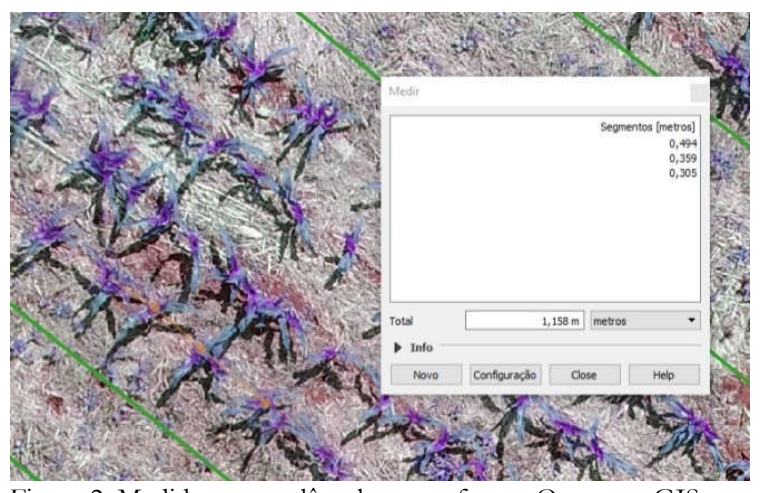

Figura 2. Medidas entre plântulas no software Quantum GIS.

Figure 2. Seedling measurements in Quantum GIS software.

Os resultados serão submetidos aos testes de Levene e Shapiro-Wilk, com o objetivo de avaliar a homogeneidade e a normalidade dos erros. Aos dados será empregada a análise de variância e as médias comparadas pelo teste de Tukey a $5 \%$ de probabilidade, utilizando o programa computacional R Core Team (2019).

\section{RESULTADOS}

A análise de variância indicou que as características espaçamento, porcentuais dos espaçamentos aceitáveis, falhos e múltiplos entre plântulas, pela avaliação por meio de imagem, da distribuição longitudinal entre plântulas não diferem entre si.

As variáveis espaçamento entre plântulas, espaçamentos aceitáveis, falhos e múltiplos não diferiram com o aumento da velocidade de trabalho do conjunto mecanizado pelo método de avaliação por imagem. A variação dos sulcadores também não interferiram na avaliação dos espaçamentos entre plântulas.

A análise de variância, pelo método manual de avaliação, indicou efeito significativo das fontes de variação velocidade de trabalho nas variáveis espaçamentos aceitáveis e falhos; bem como tipo de sulcador nas variáveis, espaçamentos aceitáveis e múltiplos, isoladamente. A interação foi significativa para velocidade de trabalho e tipo de sulcador na variável espaçamentos aceitáveis. O aumento da velocidade de trabalho influenciou o percentual de espaçamentos aceitáveis entre plântulas, utilizando o método manual, na velocidade de $6 \mathrm{~km} \mathrm{~h}^{-1}$ para a deposição da semente pelo sulcador de disco (Tabela 2).

Os espaçamentos falhos entre plântulas não foram influenciados a partir da velocidade de trabalho de $8,0 \mathrm{~km} \mathrm{~h}$ 1. Na velocidade de $6 \mathrm{~km} \mathrm{~h}^{-1}$ apresentou o menor percentual de espaçamento falho (Tabela 3 ).

O tipo de sulcador influencia os espaçamentos múltiplos entre plântulas, sendo maior quando se utiliza haste sulcadora (Tabela 4). 
Tabela 2. Porcentagem média de espaçamentos aceitáveis para o método manual de avaliação na interação velocidade de trabalho e tipo de sulcador.

Table 2. Average percentage of acceptable spacing for the manual method of evaluation in the interaction working speed and furrow type.

\begin{tabular}{ccc}
\hline & \multicolumn{2}{c}{ Selocidade } \\
\cline { 2 - 3 }$\left(\mathrm{km} \mathrm{h}^{-1}\right)$ & Disco & Haste \\
\hline 6 & $75,62 \mathrm{aA}$ & $41,87 \mathrm{aB}$ \\
8 & $61,87 \mathrm{bA}$ & $39,37 \mathrm{aB}$ \\
10 & $57,50 \mathrm{bA}$ & $41,87 \mathrm{aB}$ \\
12 & $51,87 \mathrm{bA}$ & $40,00 \mathrm{aB}$ \\
\hline
\end{tabular}

Médias seguidas de letras distintas minúsculas na coluna e maiúscula na linha diferem entre si pelo teste de Tukey $(\mathrm{p}>0,05)$.

Tabela 3. Porcentagem média de espaçamentos falhos para o método manual de avaliação em função da velocidade de trabalho. Table 3. Average percentage of acceptable flawed for the manual method of evaluation in function working speed.

\begin{tabular}{cc}
\hline $\begin{array}{c}\text { Velocidade de trabalho } \\
\left(\mathrm{km} \mathrm{h}^{-1}\right)\end{array}$ & $\begin{array}{c}\text { Espaçamentos falhos } \\
(\%)\end{array}$ \\
\hline 6 & $20,63 \mathrm{~b}$ \\
8 & $28,44 \mathrm{ab}$ \\
10 & $27,19 \mathrm{ab}$ \\
12 & $33,44 \mathrm{a}$ \\
\hline
\end{tabular}

Médias seguidas de mesma letra não diferem entre si, pelo teste de Tukey ( $\mathrm{p}$ $>0,05)$.

Tabela 4. Porcentagem média de espaçamentos múltiplos para o método manual de avaliação em função do tipo de sulcador.

Table 4. Average percentage of multiple spacing for the manual method of evaluation in function working speed and furrow type.

\begin{tabular}{cc}
\hline Sulcador & $\begin{array}{c}\text { Espaçamentos múltiplos } \\
(\%)\end{array}$ \\
\hline Disco & $11,25 \mathrm{~b}$ \\
Haste & $31,41 \mathrm{a}$ \\
\hline Médias seguidas de letras distintas diferem entre si, pelo teste $\mathrm{F}(\mathrm{p}>0,05)$.
\end{tabular}

O índice de velocidade de emergência de plântulas foi maior para o sulcador de haste (Tabela 5).

O tempo médio de emergência, em dias, foi maior para o sulcador de hastes à medida que se aumentou a velocidade. $\mathrm{O}$ aumento da velocidade de trabalho não interfere no tempo médio de emergência de plântulas ao utilizar o sulcador de disco, porém o mesmo não foi observado para o sulcador de haste (Tabela 6).

Tabela 5. Média do índice de velocidade de emergência, método manual de avaliação, para variável tipo de sulcador.

Table 5. Average emergence speed index, manual evaluation method, variable furrower type.

\begin{tabular}{cc}
\hline Sulcador & IVE \\
\hline Disco & $16,27 \mathrm{~b}$ \\
Haste & $19,18 \mathrm{a}$ \\
\hline Médias seguidas de mesma letra não diferem entre si, pelo teste $\mathrm{F}(\mathrm{p}>0,05)$.
\end{tabular}

Tabela 6. Média do tempo médio de emergência (dias), método manual de avaliação, para a interação velocidade de trabalho e tipo de sulcador.

Table 6. Average time emergence, manual evaluation method, in the interaction working speed and furrow type.

\begin{tabular}{ccc}
\hline Velocidade $\left(\mathrm{km} \mathrm{h}^{-}\right.$ & \multicolumn{2}{c}{ Sulcador } \\
\cline { 2 - 3 } 1$)$ & Disco & Haste \\
\hline 6 & $6,35 \mathrm{aA}$ & $6,22 \mathrm{bB}$ \\
8 & $6,31 \mathrm{aA}$ & $6,33 \mathrm{abA}$ \\
10 & $6,27 \mathrm{aB}$ & $6,39 \mathrm{aA}$ \\
12 & $6,37 \mathrm{aA}$ & $6,41 \mathrm{aA}$ \\
\hline
\end{tabular}

Médias seguidas de letras distintas minúsculas na coluna e maiúscula na coluna diferem entre si pelo teste de Tukey $(p>0,05)$.

\section{DISCUSSÃO}

As variáveis espaçamento entre plântulas, espaçamentos aceitáveis, falhos e múltiplos não diferiram com o aumento da velocidade de trabalho do conjunto mecanizado pelo método de avaliação por imagem.

Porém, necessita-se de mais pesquisas ou outras técnicas de processamento de imagem, a fim de maior aprofundamento na influência do método nos parâmetros em estudo, visto que se encontrou bastante dificuldade em mensurar as distâncias centro a centro entre as plântulas de milho. Pôde-se observar que a cobertura vegetal sobre o solo foi um dos fatores que mais dificultaram o contraste com as plântulas de milho, fato este não teria sido problema se tivesse sido preparo convencional do solo.

O aumento da velocidade de trabalho pode reduzir o tempo disponível para preenchimento dos alvéolos dos discos dosadores.

Ferreira et al. (2018) avaliando a distribuição longitudinal das sementes de milho sob diferentes velocidades $(3,5 ; 5,5$; $7,5 \mathrm{~km} \mathrm{~h}^{-1}$ ) na semeadura, utilizando semeadoras de precisão mecânica e pneumática afirmam que sementes dosadas pela semeadora mecânica, a maior velocidade ocasionou menor número de sementes com espaçamento aceitável. Já para a semeadora pneumática, o número de sementes aceitáveis se manteve sem diferença estatística nas três velocidades de semeadura.

Resultados semelhantes foram encontrados por Cortez et al. (2020) avaliando a semeadura do milho por meio de mecanismos mecânico e pneumático, em diferentes velocidades de trabalho; esses concluíram que o sistema pneumático promove uma melhor distribuição longitudinal de sementes, atingindo valores superiores a $90 \%$ e o incremento da velocidade reduz os espaçamentos normais entre plântulas e consequentemente aumenta-se os falhos.

A semeadura com sulcadores do tipo disco desencontrado realizada em velocidades menores que $8,0 \mathrm{~km}$ $\mathrm{h}^{-1}$, proporcionaram uma porcentagem de aceitáveis acima de $50 \%$; valor não obtido com sulcador do tipo haste. Carpes et al. (2018), avaliando a velocidade de trabalho de uma semeadora pneumática e a inclinação do disco dosador de sementes, verificaram o efeito significativo da interação dos fatores nos espaçamentos aceitáveis entre plântulas, onde o disco inclinado a $11^{\circ}$ à esquerda e nivelado reduziu os espaçamentos aceitáveis com o aumento da velocidade da semeadora.

O mecanismo sulcador de disco, independente da velocidade, apresentou maiores valores médios para espaçamento aceitáveis. A não significância para espaçamentos aceitáveis com o aumento da velocidade pode ser explicado pela capacidade do mecanismo dosador individualizar as sementes, sendo assim, as sementes foram capazes de serem distribuídas no solo com o espaçamento estabelecido. Os mecanismos sulcadores de disco apresentam a tendência de depositarem as sementes em menores profundidades e consequentemente maior velocidade de emergência e menor tempo de emergência das plântulas.

Pesquisas realizadas por Coelho (1996) apontam que valores de espaçamentos aceitáveis são considerados bons quando superiores a $60 \%$, com coeficiente de variação inferior a $50 \%$. Os valores encontrados para espaçamentos aceitáveis se enquadram nos percentuais mencionados apenas quando se utiliza o mecanismo sulcador de disco para as velocidades até $8,0 \mathrm{~km} \mathrm{~h}^{-1}$. Klein; Klein (2018) trabalhando com semeadora-adubadora de discos alveolares encontraram

Nativa, Sinop, v. 9, n. 2, p. 157-162, mar./abr. 2021. 
valores superiores a $80 \%$ de espaçamentos aceitáveis no plantio mecanizado do milho, apresentando desempenho superior aos requisitos mínimos exigidos para sua certificação.

Resultados distintos foram encontrados por Santos et al. (2016), com melhores resultados de espaçamentos aceitáveis com o uso de sulcadores do tipo haste na velocidade de trabalho de $6,3 \mathrm{~km} \mathrm{~h}^{-1}$. Jasper; Silva (2015) relatam que o mecanismo de disco duplo deve ser preferível quando o espaçamento entre fileiras é de $0,45 \mathrm{~m}$ e, o uso de haste é uma boa opção com espaçamentos de $0,9 \mathrm{~m}$, a fim de obter em ambas as situações menores custos operacionais.

$\mathrm{Na}$ menor velocidade estudada, $6 \mathrm{~km} \mathrm{~h}^{-1}$, apresentou o menor percentual de espaçamento falho, o que é esperado devido ao maior percentual de espaçamento aceitável. Estes resultados corroboram Bottega et al. (2018), que encontraram maiores percentuais de falhas na velocidade de $8,0 \mathrm{~km} \mathrm{~h}^{-1}$, quando comparada com as velocidades de 4 e $6 \mathrm{~km} \mathrm{~h}^{-1}$.

De acordo com Klein; Klein (2018), as maiores produções de massa de grãos por espiga foram obtidas para os espaçamentos falhos, enquanto nos espaçamentos aceitáveis e duplos não foram observadas diferenças na produção de massa de grãos por espiga.

Relacionando os fatores distribuição de sementes pelo sistema mecânico e pneumático com a produtividade da cultura do milho, nas velocidades de 3,0; 5,0; 7,0 e 9,0 $\mathrm{km} \mathrm{h}^{-}$ ${ }^{1}$, Cortez et al. (2020), obtiveram maiores rendimentos na velocidade de $5,0 \mathrm{~km} \mathrm{~h}^{-1}$ com o sistema mecânico, quando os dados de produtividade foram agrupados em classes.

A patinagem das rodas motrizes do trator é maior quando se realiza a abertura do sulco com órgãos ativos fixos, ou seja, sem movimento rotativo, como no caso da haste sulcadora, com requerimento de força de tração $22,28 \%$ superior ao solicitado pelo mecanismo rotativo (FRANCETTO et al. 2015).

Este fato ajuda a explicar maiores espaçamentos múltiplos para o mecanismo de haste, com possível condução das sementes enquanto a semeadora estava com o deslocamento limitado, pela ação da patinagem das rodas motrizes do trator. Portanto, pode ter sido depositado mais sementes em um menor intervalo de tempo, aumentando assim os espaçamentos múltiplos entre plântulas.

A porcentagem de múltiplos foi 179,2\% maior quando utilizou o sulcador do tipo haste. A análise de variância das características índice de velocidade de emergência - IVE e tempo médio de emergência - TM, pelo método manual de avaliação da distribuição longitudinal, demonstrou que a velocidade de trabalho não influenciou o IVE de plântulas, possivelmente devido à uniformidade na profundidade de deposição no sulco de semeadura e também não provocou danos à semente, mesmo se tratando de uma semeadora onde a individualização das sementes é mecânica, ou seja, com dosador de disco horizontal perfurado. Por outro lado, Bottega et al. (2018) observaram que o valor médio de IVE para o dosador pneumático foi maior e diferiu estatisticamente para o dosador de disco. Portanto, as sementes não perderam seu vigor, com isso, não alterou sua velocidade de emergência.

O índice de velocidade de emergência de plântulas ter sido maior para o sulcador de haste pode ser explicado pela tendência a aprofundar mais no solo e depositar a semente em maiores profundidades, mesmo tendo feito testes de regulagem e verificação da profundidade de deposição das mesmas no solo. Portanto, pequenos acréscimos da profundidade de colocação das sementes com o uso da haste sulcadora acarretaram em aumento no índice de emergência.

Ao se realizar o sulco com discos pode-se trabalhar em maiores velocidades que não interferirá no tempo de emergência das plântulas de milho; porém, ao se trabalhar com haste é preferível optar por velocidades até $8,0 \mathrm{~km} \mathrm{~h}^{-1}$.

\section{CONCLUSÕES}

O conjunto mecanizado operando a $6,0 \mathrm{~km} \mathrm{~h}^{-1}$, com sulcador do tipo disco, promove o melhor estabelecimento inicial da cultura do milho.

As plântulas de milho emergem em menor tempo nas velocidades até $8,0 \mathrm{~km} \mathrm{~h}^{-1}$ com sulcador de disco.

A metodologia de uso de imagens captadas por aeronave remotamente pilotada não se mostrou eficaz para a determinação da distribuição longitudinal de plântulas, necessitando de novas pesquisas.

\section{AGRADECIMENTOS}

Os autores agradecem à Fundação de Amparo à Pesquisa de Minais Gerais, FAPEMIG, pelo apoio financeiro.

\section{REFERÊNCIAS}

ARANTES, B. H. T.; ARANTES, L. T.; COSTA, E. M.; VENTURA, M. V. A. Drone aplicado na agricultura digital. Ipê Agronomic Journal, Goianésia, v. 3, n. 1, p. 14-18, 2019.

BOTTEGA, E. L.; VIAN, T.; GUERRA, N.; OLIVEIRA NETO, A. M. de. Diferentes dosadores de sementes e velocidades de deslocamento na semeadura do milho em plantio direto. Pesquisa Agropecuária Pernambucana, Recife, v. 22, e201707, 2018. DOI: https://doi.org/10.12661/pap.2017.014

CARPES, D. P.; ALONÇO, A. dos S.; FRANCETTO, T. R.; MOREIRA, A. R.; CHAGAS, G. S. Qualidade da distribuição longitudinal de sementes de milho por um dosador-apanhador com auxílio pneumático. Revista Engenharia na Agricultura, v. 26, n. 1, p. 43-51, 2018. DOI: https://doi.org/10.13083/reveng.v26i1.850

CARPES, D. P.; ALONÇO, A. S.; ROSSATO, F. P.; VEIT, A. A.; SOUZA, L. B.; FRANCETTO, T.R. Effect of different conductor tubes on the longitudinal distribution of corn seeds. Revista Brasileira de Engenharia Agrícola e Ambiental, Campina Grande, v. 21, n. 9, p. 1807-1929, 2017. DOI:

https://doi.org/10.1590/1807-

1929/agriambi.v21n9p657-662

COELHO, J. L. D. Ensaio e certificação das máquinas para semeadura. In: MIALHE, L. G. Máquinas agrícolas: ensaios e certificação. Piracicaba: Fealq, 1996. p. 551-570.

CORTEZ, J. W.; ANGHINONI, M.; ARCOVERDE, S. N. S. Seed metering mechanisms and tractor-seeder forward speed on corn agronomic components. Engenharia Agrícola, Jaboticabal, v. 40, n. 1, p. 61-68, 2020. DOI: http:/ /dx.doi.org/10.1590/1809-4430Eng.Agric.v40n1 p61-68/2020

EDMOND, J. B.; DRAPALA, W. L. The effects of temperature, sand and soil, and acetone on germination of okra seed. Journal of the American Society for Horticultural Science, Geneva, v. 71, p. 428-34, 1958.

FERREIRA, F. M.; OSS, L. L.; CARNEIRO, M. de A; LITTER, F. A. Distribuição longitudinal na semeadura 
do milho com semeadoras de precisão mecânica e pneumática. Nativa, Sinop, v. 7, n. 3, p. 296-300, 2019. DOI: http://dx.doi.org/10.31413/nativa.v7i3.7553

FRANCETTO, T. R.; ALONÇO, A. dos S.; BELLÉ, M. P.; FRANCK, C. J.; CARPES, D. P. Comportamento operacional de associações entre sulcadores e discos de corte para sistema de semeadura direta. Engenharia Agrícola, Jaboticabal, v. 35, n. 3, p. 542-554, 2015. DOI: http://dx.doi.org/10.1590/1809-4430-

Eng.Agric.v35n3p542-554/2015

ISO_International Organization for Standardization. (ISO) 7256/1: Sowing equipment -test methods: part 1. Single seed drills (precision drills). Geneva: ISO, 1984. 14 p.

JASPER, S. P.; SILVA, P. R. A. da. Comparação econômica de diferentes mecanismos sulcadores de semeadoras em plantio direto de milho. Nucleus, Ituverava, v. 12, n. 1, p. 181-188, 2015.2 DOI: http://dx.doi.org/10.3738/1982.2278.1185

KLEIN, V. A.; KLEIN, C. Metodologia de avaliação da distribuição das plantas de milho no campo versus massa de grãos. Pesquisa Agropecuária Pernanbucana, Recife, v. 22, p. 1-6, 2018. DOI: https://doi.org/10.12661/pap.2017.010

KOPPEN, W. Climatologia com un estudio de los climas de la tierra. México: Fondo de Cultura Econômica, 1948. $478 \mathrm{p}$.

MAGUIRE, J. D. Speed of germination-aid in selection and evaluation for seedling emergence and vigor. Crop Science, Madison, v. 2, n. 1, p. 176-177, 1962.

R Core Team (2019). R: A language and environment for statistical computing. R Foundation for Statistical Comp uting, Vienna, Austria. Disponível em: $<$ https://www.R-project.org/>. Acesso em: 19 jun. 2019.

SANTOS, V. C. dos; SANTOS, P. R. A. dos; LIMA, A. O.; PEREIRA, V. R. F.; GONÇALVES, F. R. F.; CHIODEROLI, C. A. Performance of a seeder according to the displacement velocity and furrowing mechanism for fertilizer deposition. Revista Brasileira de Engenharia Agrícola e Ambiental, Campina Grande, v. 20, n. 3, p. 286-291, 2016. DOI: http://dx.doi.org/10.1590/18071929/agriambi.v20n3p286-291

STOLF, R.; GARCIA, T. B.; NERIS, L. de O.; TRINDADE JÚNIOR, O.; REICHARDT, K. Avaliação de falhas em cana-de-açúcar segundo o método de Stolf utilizando imagens aéreas de alta precisão obtidas por Vant. Stab, Piracicaba, v. 34, n. 4, p. 32-39, 2016.

ORMOND, A. T. S.; FURLANI, C. E. A.; OLIVEIRA, M. F. de; NOROHA, R. H. de F.; TAVARES, T. de O.; MENEZES, P. C. de. Maize sowing speeds and seed metering mechanisms. Journal of Agricultural Science, Beaver Cree, v. 10, n. 9, p. 468-476, 2018. DOI: https://doi.org/10.5539/jas.v10n9p468 